\title{
The efficacy and safety of intravenous chlorpromazine treatment for sleep disturbance in patients with incurable cancer, with oral administration difficulty: a 1-week, prospective observational study
}

\author{
Ryohei Fujii $^{1 \#}$, Hideaki Hasuo ${ }^{2 \#}$, Hiroko Sakuma ${ }^{2}$, Miyuki Okada ${ }^{1}$, Kazuki Uchitani ${ }^{1}$ \\ ${ }^{1}$ Department of Pharmacist, Kansai Medical University Hospital, Osaka, Japan; ${ }^{2}$ Department of Psychosomatic Medicine, Kansai Medical University, \\ Osaka, Japan \\ Contributions: (I) Conception and design: R Fujii, H Hasuo; (II) Administrative support: R Fujii, H Hasuo; (III) Provision of study materials or \\ patients: All authors; (IV) Collection and assembly of data: R Fujii, H Hasuo, H Sakuma; (V) Data analysis and interpretation: R Fujii, M Okada; (VI) \\ Manuscript writing: All authors; (VII) Final approval of manuscript: All authors. \\ \#These authors contributed equally to this work. \\ Correspondence to: Hideaki Hasuo. Department of Psychosomatic Medicine, Kansai Medical University, Shinmachi 2-5-1, Hirakata, Osaka 573-1090, \\ Japan. Email: hasuohid@hirakata.kmu.ac.jp.
}

Background Sleep disturbance is a common psychiatric disorder in patients with cancer. However, many
patients with incurable cancer have difficulty receiving oral administrations, which limits treatment options
during disease progression. The aim of the present study was to assess the efficacy and safety of intravenous
chlorpromazine treatment for sleep disturbances in patients with incurable cancer, with oral administration
difficulty.

Methods: A prospective observational study was conducted among 52 patients with incurable cancer, with oral administration difficulty received daily intravenous chlorpromazine treatment for sleep disturbance from 2018 to 2020 at a single-unit university hospital. St. Mary's Hospital Sleep Questionnaire (SMHSQ) compared sleep before and after intravenous chlorpromazine administration. The primary endpoint was the efficacy rate of sleep quality [defined as a score of $\geq 4$ (range, 1-6)] 7 days after receiving chlorpromazine.

Results: Beginning the day after receiving chlorpromazine, sleep quality significantly improved from a mean score of $1.6 \pm 0.7$ to $4.3 \pm 1.2$, and $80.8 \%$ [95\% confidence interval (CI): $66.5-89.1 \%$ ] and $69.2 \%$ (95\% CI: $53.8-79.6 \%$ ) of patients reported good sleep quality 3 and 7 days after receiving chlorpromazine, respectively. The patients reported increased total sleep time and fewer awakenings during sleep, and satisfaction with sleep and difficulty falling asleep improved. Some adverse events occurred [akathisia $(\mathrm{n}=2)$, dry mouth $(\mathrm{n}=2)$, and somnolence $(\mathrm{n}=3)$ ]; all were Grade 1 (CTCAE ver5.0) and improved with chlorpromazine discontinuation. Systolic blood pressure and heart rate displayed no clinically problematic changes.

Conclusions: Intravenous chlorpromazine has a high tolerability and effectively treats sleep disturbances in patients with incurable cancer with oral administration difficulties.

Keywords: Chlorpromazine; sleep disturbance; cancer patient; intravenous; St. Mary's Hospital Sleep Questionnaire (SMHSQ)

Submitted Apr 23, 2021. Accepted for publication Jul 02, 2021.

doi: 10.21037/apm-21-948

View this article at: https://dx.doi.org/10.21037/apm-21-948 


\section{Introduction}

Sleep disturbances are experienced by many patients with cancer, including $23-70 \%$ of patients with incurable cancer (1). Sleep disturbances are a distressing symptom for patients with cancer and decrease their quality of life (QOL) (2). Additionally, sleep disturbances in patients with incurable cancer contribute to delirium.

Pharmacotherapy is one of the main treatment options to improve decreased QOL in patients with cancer due to sleep disturbances (3). However, without randomized trials for cancer-related sleep disturbances, the use of sleep medications is recommended based on the treatment of primary sleep disturbances (4). Many patients with incurable cancer have difficulty with oral administration as their disease progresses; thus, they have a limited choice of antipsychotics for parenteral administration.

Benzodiazepines have been empirically administered intravenously; however, they are associated with problems such as the development of delirium, respiratory depression, and tolerance (5-7). In particular, delirium has been reported to occur in $42-88 \%$ of patients with incurable cancer (8). There is no specific drug for sleep disturbances in patients with incurable cancer, and the best drug should be selected according to each patient's condition (9).

Intravenous chlorpromazine has been used empirically as supportive care for patients with cancer, with severe delirium and sleep disturbance $(10,11)$. Chlorpromazine is characterized by its strong sedative effects including histamine $\mathrm{H} 1$ receptor and alpha1 adrenergic receptor blockades $(12,13)$. For this reason, it does not require continuous intravenous infusion and thus significantly reduces the patients' burden compared with midazolam, which requires continuous administration.

Intravenous chlorpromazine has been used off-label for the treatment of delirium and sleep disturbances; and it has been reported that $13 \%$ of the off-label use in one acute palliative care unit was intravenous chlorpromazine (14). To our knowledge, our previous study is the only report on the efficacy of intravenous chlorpromazine against sleep disturbances in patients with cancer, or other patient populations (15).

Our previous study showed a $63.0 \%$ efficacy rate 3 days after intravenous chlorpromazine treatment for sleep disturbance in patients with incurable cancer, with oral administration difficulty, which suggests that intravenous chlorpromazine may be a short-term treatment option for sleep disturbances in patients with incurable cancer. However, despite 28 patients (93.3\%) continuing treatment after day 3, the feasibility of medium-term or long-term treatment could not be determined given the study's observational and retrospective nature, missing data, and other factors. Conversely, if a prospective observational study shows high feasibility 7 days after the chlorpromazine dose, it may be useful for providing more clinically relevant pharmacotherapy options for treating sleep disturbances.

We present the following article in accordance with the STROBE reporting checklist (available at https://dx.doi. org/10.21037/apm-21-948).

\section{Methods}

\section{Objective}

The primary objective of this study was to reassess the efficacy and safety of intravenous chlorpromazine treatment for sleep disturbances in patients with incurable cancer, with oral administration difficulty and to determine the feasibility 7 days after the dose.

\section{Study design}

This was a single-center prospective observational study conducted at a university hospital located in the largest metropolitan area in western Japan. The study subjects' data were extracted from medical records and included age, sex, primary cancer site, Eastern Cooperative Oncology Group performance status (ECOG PS), blood pressure, heart rate, psychotropic use before chlorpromazine treatment, cause of difficulty in oral administration, chlorpromazine dose, days to live after chlorpromazine treatment, adverse events [e.g., falls, delirium, extrapyramidal symptoms (EPS), vasculitis and subcutaneous induration at intravenous drip site], and assessments of sleep with the St. Mary's Hospital Sleep Questionnaire (SMHSQ) immediately before and 1, 3 , and 7 days after the dose. Three physicians specializing in palliative care with more than 15 years of experience diagnosed sleep disturbances using the American Psychiatric Association's diagnostic criteria (DSM-V) and treated the patients with intravenous chlorpromazine. In addition, the presence or absence of delirium between day 0 (pre-dose) and days 3 and 7 post-dose was diagnosed using DSM-V.

This study was conducted in accordance with the Declaration of Helsinki (as revised in 2013) and received approval from the Medical Ethics Committee of Kansai Medical University, Japan (reference number: 2017323). Informed consent was obtained from all study participants. 
This study was registered at the University Hospital Medical Information Network Clinical Trials Registry (approval number: UMIN000043869) on April 8, 2021 (registered retrospectively).

\section{Outcomes}

The primary endpoint was the efficacy rate of the SMHSQ on sleep quality 7 days after the intravenous chlorpromazine dose. The secondary endpoints were as follows: (I) before/ after comparison of hours of total sleep and hours of sleep latency; (II) before/after comparisons of the scores for the number of awakenings during sleep time, clear-headedness on arising, satisfaction with sleep, difficulty falling asleep, and difficulty falling asleep again; and (III) evaluation of adverse events. These endpoints were assessed with the SMHSQ 3 and 7 days after the treatment.

\section{Study participants}

Inclusion criteria of the study were as follows: patients with incurable cancer, with oral administration difficulty who were diagnosed with sleep disturbances; informed consent for intravenous chlorpromazine was obtained from these patients. Exclusion criteria were as follows: patients $<20$ years of age, secondary insomnia attributed to delirium or medications, or patients with psychiatric disorder with communication difficulty, such as dementia. This study was conducted at Kansai Medical University Hospital from May 2018 to December 2020. A total of 1,919 patients with cancer who visited the Department of Palliative Care during this period were consecutively enrolled in this study. Among them, 73 met the inclusion criteria, and of these, 21 met the exclusion criteria. A final total of 52 patients with cancer were included in the study. In this study, compounding cardiac risks were also considered as exclusion criteria, but the patient was not applicable.

We have empirically administered intravenous chlorpromazine at bedtime for the relief of sleep disturbances in patients with oral administration difficulty, who ware benzodiazepines refractory or intolerant. The initial dose of intravenous chlorpromazine was $7.5-25 \mathrm{mg}$ /day with an administration rate of $25 \mathrm{mg} / \mathrm{h}$ and was administered at $\sim 9 \mathrm{pm}$ and adjusted depending on the patient's symptoms.

\section{SMHSQ}

The SMHSQ is a tool designed to assess sleep problems in hospitalized patients (16). The focus is on subjective assessments of sleep quality, with assessments of the last 24 hours of sleep. This self-administered questionnaire has been shown to be reliable enough for use in psychiatric and medical inpatients (17). The SMHSQ consists of a total of 14 questions, typical of which are sleep quality (range, 1-6), hours of total sleep, hours of sleep latency, number of awakenings during sleep time (range, 0-7), satisfaction with sleep (range, 1-5), difficulty falling asleep (range, $1-4)$, difficulty falling asleep again (range, 1-2), and clearheadedness on arising (range, 1-6). Low scores, indicate more disturbed sleep. Subjects in this study completed the SMHSQ through interviews by the trained nurse at the time of awakening, at pre-dose on the dosing day (day 0 ) and days 1,3 , and 7 post-dose.

\section{Efficacy of intravenous chlorpromazine}

The primary endpoint was sleep quality, in agreement with previous studies $(18,19)$. Secondary endpoints included total sleep time, sleep latency time, number of awakenings during sleep time, satisfaction with sleep, difficulty falling asleep, difficulty falling asleep again, and clear-headedness on arising. Sleep quality was assessed based on a comparison between pre-dose and post-dose scores and the mean chlorpromazine efficacy rate on day 7 . The efficacy rate of chlorpromazine is defined as the number of patients with a score of $\geq 4$ based on answers to sleep quality questions in the SMHSQ ( $1=$ very poor, 2 = poor, $3=$ fairly poor, $4=$ fairly good, $5=$ good, and $6=$ very good).

\section{Safety of intravenous chlorpromazine}

Adverse events were assessed according to the Common Terminology Criteria for Adverse Events (CTCAE) Version 5.0 established by the National Cancer Institute. The presence or absence of EPS was assessed, using the EPS rating scale developed to assess drug-induced movement disorders (20). The presence or absence of delirium as a typical adverse event was assessed by a psycho-oncology expert, using DSM-V. Hemodynamic effects were assessed by comparing blood pressure and heart rate between day 0 (pre-dose) and days 3 and 7 post-dose, and the presence or absence of clinical adverse episodes during intravenous administration. Potential vascular or skin lesions around the site of administration were assessed based on the presence or absence of vasculitis and subcutaneous callus. 


\section{Sample size calculation}

Forty-four evaluable patients would provide an $80 \%$ power to detect an effect size as small as 0.5 using a two-sided paired $t$-test with a significance level of $5 \%$ to compare the change in sleep quality. The required sample size was 50 , given an estimated dropout rate of $10 \%$.

\section{Statistical analysis}

The main analysis was performed for patients who used chlorpromazine more than once according to the intentionto-treat principle, and all data were reported as means with 95\% confidence intervals (CIs), ranges, average or median values, or frequencies (\%), as appropriate. We calculated the 95\% CIs of the patients with scores of $\geq 4$ based on answers to sleep quality questions in the SMHSQ, and we compared the changes in the chlorpromazine efficacy rate (days $0,1,3$, and 7) using Fisher's exact test. Each continuous variable of the SMHSQ, blood pressure, and heart rate, was compared using Wilcoxon signed-rank test between pre-dose and postdose time points. For missing values, we applied the last observation carried forward method and confirmed the same result using the worst observation carried forward method. We assumed statistical significance at 0.05 . We decided not to adjust for multiple comparisons because of the completely exploratory nature of this study. All analyses were performed using JMP ${ }^{\circledR}$ 14.2.0 (SAS Institute Inc., Cary, NC, USA).

\section{Results}

The demographic and clinical characteristics of the 52 study subjects are shown in Table 1. The median subject age was 65.5 (range, 41-92) years. The mean performance status was $2.6 \pm 1.0$. The median survival period after chlorpromazine treatment was 34.5 (range, 1-790) days. All patients received intravenous chlorpromazine at a dose of $13.3 \pm 4.3$ (range, $7.5-25) \mathrm{mg} / \mathrm{day}, 0.26 \pm 0.11$ (range, $0.11-0.61) \mathrm{mg} / \mathrm{kg}$. Study discontinuation occurred for the following reasons: 7 adverse events (13.5\%), 6 deaths (11.5\%), 4 patients' wishes $(7.7 \%)$, and 1 transfer to another hospital (1.9\%). A total of 17 patients $(32.7 \%)$ used opioid from chlorpromazine predose; however, opioid was not adjusted or switched during the chlorpromazine treatment.

\section{Effectiveness of chlorpromazine}

Sleep quality significantly improved from a mean score of $1.6 \pm 0.7$ to $4.3 \pm 1.2$ on day $1(\mathrm{P}<0.0001)$ (Figure 1$)$. No patients had sleep quality scores of $\geq 4$ before intravenous chlorpromazine. Furthermore, $80.8 \%$ (95\% CI: $66.5-$ $89.1 \%$ ) and $69.2 \%$ (95\% CI: $53.8-79.6 \%)$ of patients had good sleep quality on days 3 and 7 , respectively. The patients reported increased hours of total sleep, fewer awakenings during sleep time, and improvements for hours of sleep latency, clear-headedness on arising, satisfaction with sleep, difficulty falling asleep, and difficulty falling asleep again $(\mathrm{P}<0.0001)$. Three patients $(5.8 \%)$ only took chlorpromazine once, and reasons for not continuing were two adverse events and one death. In addition, no patients improved to a score of $\geq 4$ to sleep quality on day 1 . No significant effect of opioid was observed on sleep quality on days $3(\mathrm{P}=0.0616)$ and $7(\mathrm{P}=1.0000)$.

\section{Safety of intravenous chlorpromazine}

Six patients (11.5\%) died during chlorpromazine treatment; however, these deaths were due to disease progression and were not causally related to chlorpromazine. A causal relationship with chlorpromazine could not be ruled out for 7 adverse events (13.5\%) that occurred within 3 days after chlorpromazine treatment: two events of akathisia, two events of dry mouth, and three events of somnolence. All adverse events were Grade 1 (CTCAE) and improved with treatment discontinuation. The two acute cases of akathisia included a 79-year-old male patient with a history of EPS who developed akathisia on day 1 and a 47-year-old male patient who developed akathisia on day 2. No falls, delirium, vasculitis, or subcutaneous induration at the intravenous site occurred after chlorpromazine treatment.

No significant effect of chlorpromazine was observed on any vital signs (systolic blood pressure, heart rate). Systolic blood pressure did not differ among days 0,3 , and 7 $[124.2 \pm 21.3,119.8 \pm 24.4(\mathrm{P}=0.0819)$, and $120.7 \pm 25.0 \mathrm{mmHg}$ $(\mathrm{P}=0.1494)$, respectively]. Similarly, heart rate did not differ among days 0,3 , and $7[91.8 \pm 14.6,96.0 \pm 19.9(\mathrm{P}=0.0765)$, and $96.8 \pm 22.3 \mathrm{bpm}(\mathrm{P}=0.0639)$, respectively]. However, one patient developed paroxysmal supraventricular tachycardia (PSVT) on day 4. According to the cardiologist, hypotension due to dehydration after ascites drainage was the cause of PSVT. Given the lack of a causal relationship with chlorpromazine, chlorpromazine administration could be continued in that patient. No clinically important episodes resulting in discontinuation occurred during the intravenous chlorpromazine treatment. 
Table 1 Clinical characteristics of study subjects at the time of enrolment

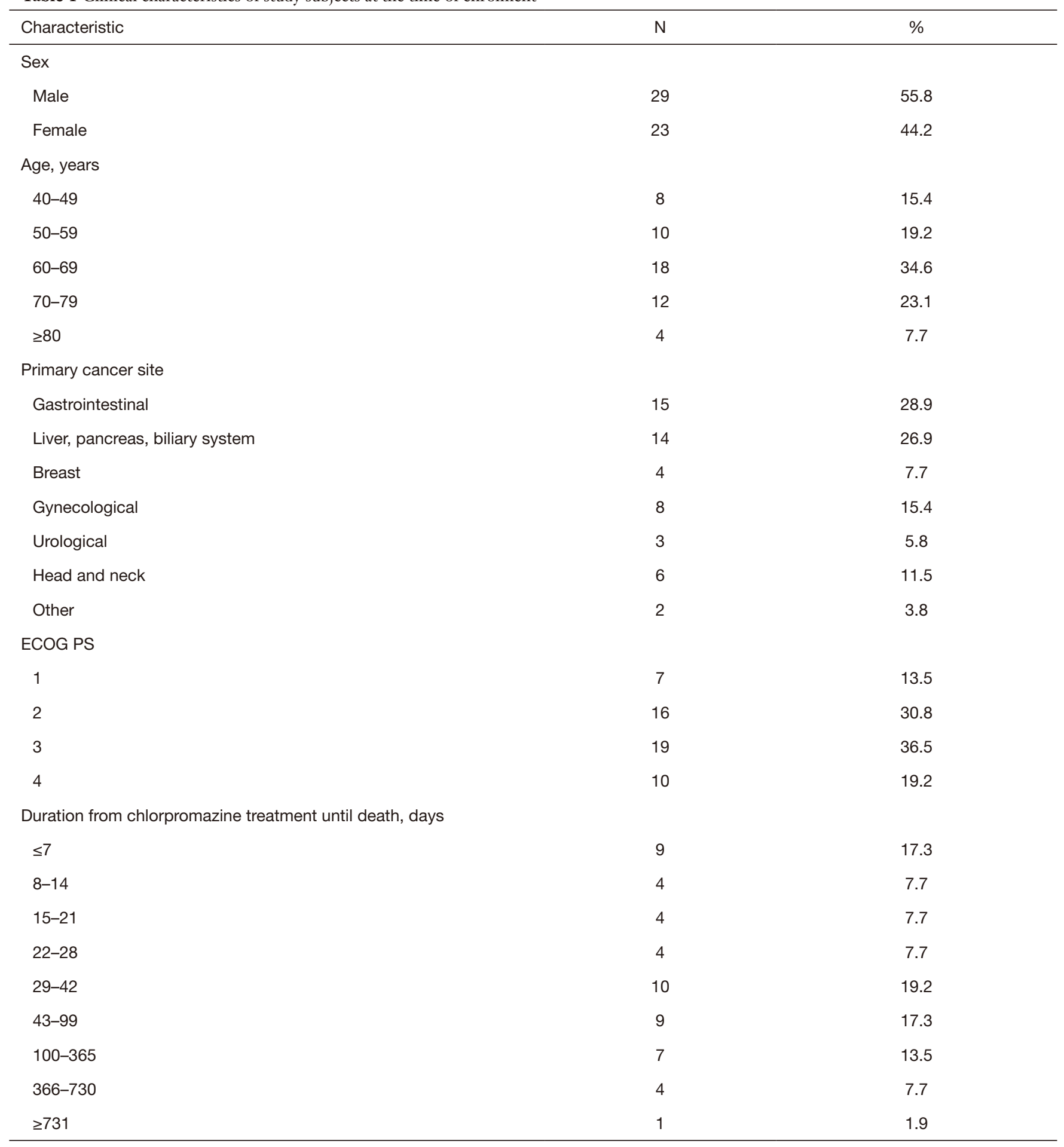

Table 1 (continued) 
Table 1 (continued)

\begin{tabular}{|c|c|c|}
\hline Characteristic & $\mathrm{N}$ & $\%$ \\
\hline Severe prostration & 7 & 13.5 \\
\hline Dysphagia & 12 & 23.1 \\
\hline Gastrointestinal obstruction & 16 & 30.8 \\
\hline Other & 10 & 19.2 \\
\hline \multicolumn{3}{|l|}{ Psychotropic use before chlorpromazine treatment } \\
\hline Nonbenzodiazepine PO & 1 & 1.9 \\
\hline Benzodiazepine PO or IV & 5 & 9.7 \\
\hline Suvorexant PO & 4 & 7.7 \\
\hline Multi-acting receptor targeted antipsychotics PO & 4 & 7.7 \\
\hline Haloperidol IV & 9 & 17.3 \\
\hline Haloperidol IV + suvorexant PO & 1 & 1.9 \\
\hline No use & 26 & 50.0 \\
\hline
\end{tabular}

ECOG PS, Eastern Cooperative Oncology Group performance status; PO, per os; IV, intravenous.

\section{Discussion}

This is, to our knowledge, the first study to prospectively assess the efficacy and safety of intravenous chlorpromazine for sleep disturbances in patients with incurable cancer, with oral administration difficulty and the first study to clarify a high feasibility of this treatment on day 7.

Notably, this study clarified the degree of sleep quality improvement in patients with incurable cancer, with oral administration difficulty who received intravenous chlorpromazine. A high efficacy rate of $69.2 \%$ was reported on day 7.

The efficacy rate on day 3 was $80.8 \%$, which was higher than the $63.0 \%$ reported in a previous study (15). Patients with cancer with a prognosis of less than 2 months were reported to have more severe sleep disturbances than those with a prognosis of 3 to 5 months (21). Thus, the shorter the prognosis, the greater the extent of intractable sleep disturbances. The mean survival time after chlorpromazine treatment in the previous study was $28.7 \pm 16.8$ days, which was shorter than the $110.1 \pm 182.6$ days in this study. That difference may contribute to the difference in efficacy rates.
The efficacy rate on day 7 (69.2\%) could be considered relatively high; however, it could not be compared with previous studies given the lack of relevant studies on sleep disturbances in patients with cancer or other patient populations. According to the American Academy of Sleep Medicine's practice guidelines, the threshold for change in total sleep time is considered clinically meaningful when the subjective total sleep time increases by more than 30 minutes, and the threshold for the amount of change in sleep onset latency is considered clinically meaningful when the subjective sleep onset latency is reduced by more than 20 minutes (22). In this study, the percentages of patients whose subjective total sleep time was prolonged by more than 30 minutes and whose subjective sleep onset latency was shortened by more than 20 minutes at each evaluation time point, which were defined as clinical improvement, are shown in Table 2. In both cases, clinical improvement was observed from day 1 compared with day 0 . Intravenous chlorpromazine can cover the normal sleep time, because the mean residence time of 8.88 hours irrespective of the dose level $(12,13)$. Therefore, intravenous chlorpromazine has both quick-acting and long-lasting effects on sleep 


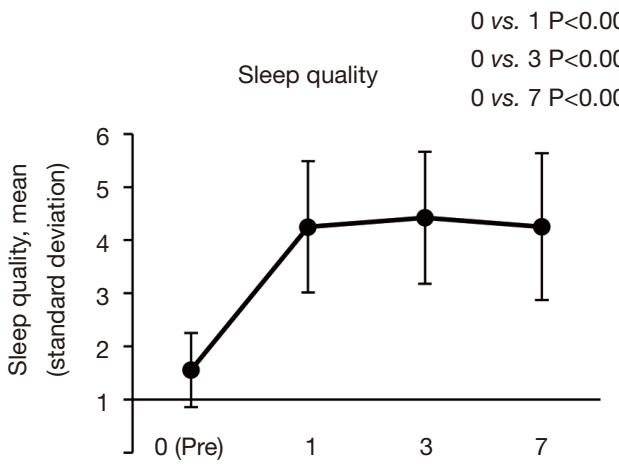

Days after intravenous chlorpromazine

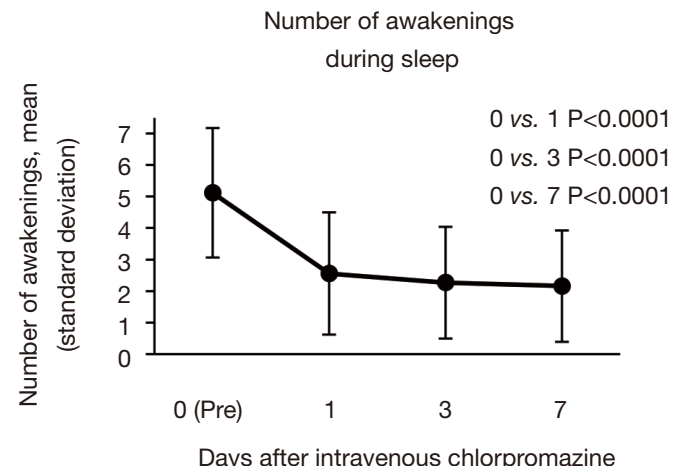

Days after intravenous chlorpromazine

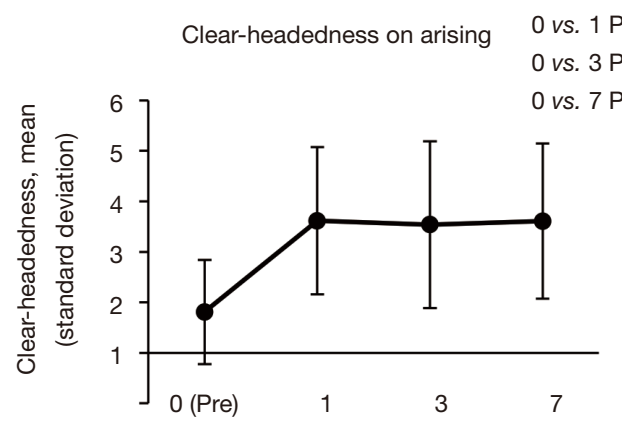

Days after intravenous chlorpromazine

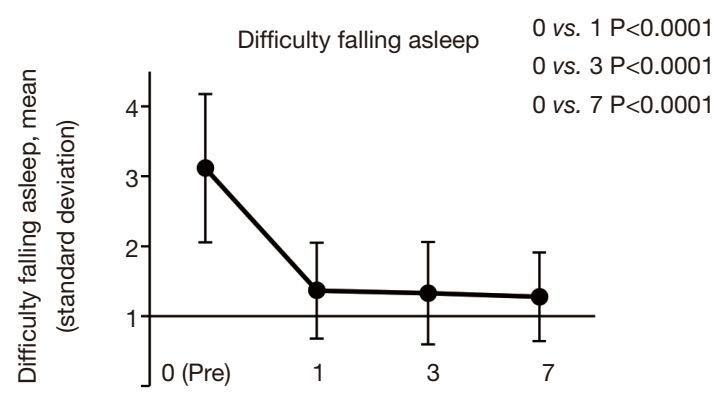

Days after intravenous chlorpromazine
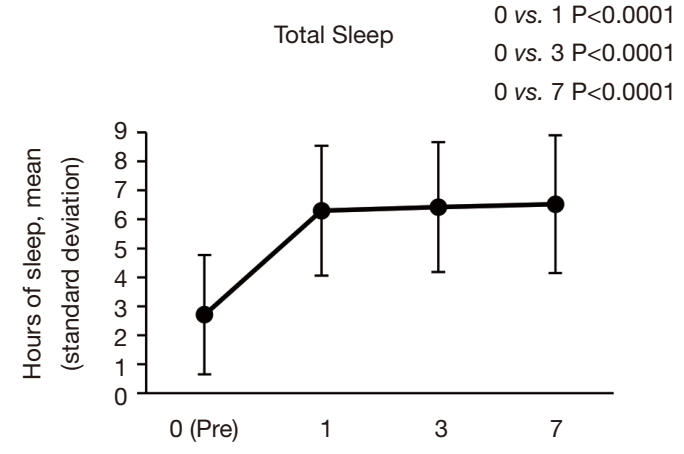

Days after intravenous chlorpromazine
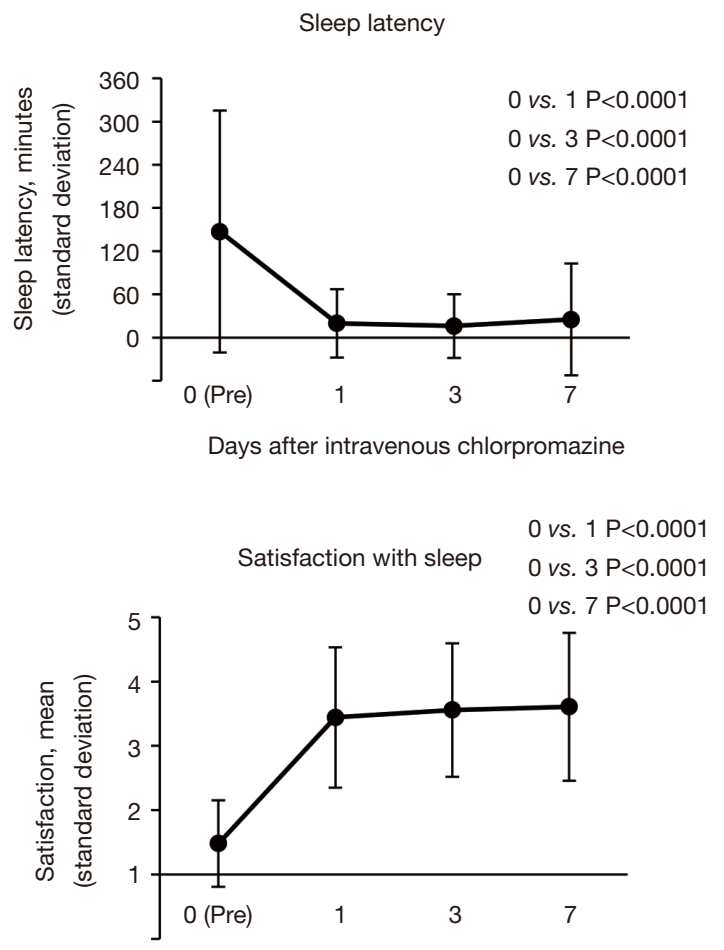

Days after intravenous chlorpromazine

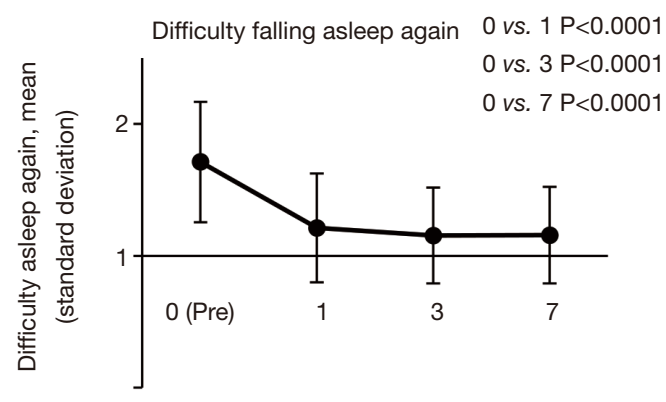

Days after intravenous chlorpromazine

Figure 1 Change in mean scores based on SMHSQ over the first 7 days after treatment. SMHSQ, St. Mary's Hospital Sleep Questionnaire. 
Table 2 Efficacy rate and change in mean scores based on SMHSQ over the first 7 days after treatment

\begin{tabular}{|c|c|c|c|c|c|}
\hline Characteristic & \multicolumn{4}{|c|}{ Days after intravenous chlorpromazine } & $\mathrm{P}$ \\
\hline \multicolumn{6}{|l|}{ Primary end point } \\
\hline Efficacy rate of sleep quality ${ }^{\dagger}(\%)$ & - & 76.9 & 80.8 & 69.2 & $<0.0001$ \\
\hline $95 \% \mathrm{Cl}(\mathrm{n}=52)$ & - & $62.1,86.0$ & $66.5,89.1$ & $53.8,79.6$ & \\
\hline SD & 0.7 & 1.2 & 1.2 & 1.4 & \\
\hline \multicolumn{6}{|l|}{ Secondary end points } \\
\hline Efficacy rate of total sleep $p^{\ddagger}(\%)$ & - & 96.2 & 90.4 & 88.5 & $<0.0001$ \\
\hline $95 \% \mathrm{Cl}(\mathrm{n}=52)$ & - & $85.5,99.7$ & $77.9,96.2$ & $75.5,94.8$ & \\
\hline Efficacy rate of sleep latency ${ }^{\S}(\%)$ & - & 69.2 & 71.2 & 76.9 & $<0.0001$ \\
\hline $95 \% \mathrm{Cl}(\mathrm{n}=52)$ & - & $53.8,79.6$ & $55.8,81.2$ & $62.1,86.0$ & \\
\hline Sleep latency (min) & 147.1 & 19.8 & 16.1 & 25.0 & $<0.0001$ \\
\hline SD & 168.1 & 47.4 & 44.2 & 77.6 & \\
\hline
\end{tabular}

${ }^{\dagger}$, the efficacy rate of chlorpromazine is defined as the number of patients with a score of $\geq 4$ based on answers to sleep quality questions in the SMHSQ; ${ }^{\ddagger}$, the efficacy rate of chlorpromazine is defined as the number of patients with total sleep time prolonged by more than 30 minutes; ${ }^{\S}$, the efficacy rate of chlorpromazine is defined as the number of patients with sleep latency time shortened by more than 20 minutes. SMHSQ, St. Mary's Hospital Sleep Questionnaire; Cl, confidence interval; SD, standard deviation.

quality, contributing to both shortening sleep latency time and lengthening total sleep time. Taken together, the efficacy of intravenous chlorpromazine for treating sleep disturbance in patients with incurable cancer, with oral administration difficulty can be expected in the medium term during the administration period.

The second and most important finding was the clarification of the high safety of intravenous chlorpromazine for use in patients with incurable cancer, with oral administration difficulty.

Adverse events often associated with chlorpromazine treatment, including falls, and effects on circulation dynamics such as vasculitis were not observed throughout this study. However, there were two events of akathisia, two events of dry mouth, and three events of somnolence. Akathisia is among the most common adverse drug effect patients experience from dopamine-receptor blocking agents. In schizophrenic patients, the incidence of akathisia was $31.3 \%$ with a mean dose of $328 \mathrm{mg} /$ day of chlorpromazine equivalent (23). The higher the antipsychotic dose, the stronger the degree and the higher the frequency of akathisia (24). Consequently, it is not possible to make a simple comparison, although the frequency was low (3.9\%) in this study. Acute akathisia usually occurs within 4 weeks after starting or increasing the antipsychotic dose (25), and a history of EPS and the male gender are risk factors for EPS $(26,27)$, which were similar to this study. In the two events of dry mouth, the anticholinergic effect of chlorpromazine was a direct cause of the event onset. The three somnolence events showed improvements in sleep quality due to the sedative effect of chlorpromazine by blocking histamine $\mathrm{H} 1$ and alpha 1 adrenergic receptors. However, excessive sedation may have been a direct cause of somnolence, suggesting that the treatment could have been continued by reducing the chlorpromazine dose.

By contrast, no instances of delirium, which often occurs with pharmacotherapy for sleep disorders, were noted in this study. Delirium is also said to be induced by chlorpromazine, and the dose level inducing delirium was reported to be $40 \mathrm{mg} /$ day of intravenous chlorpromazine in advanced patients with cancer and $36-50 \mathrm{mg} /$ day of 
intramuscular chlorpromazine in patients with hospitalized acquired immunodeficiency syndrome $(10,14)$. The low dose used in this study $(13.3 \mathrm{mg} /$ day $)$ may have prevented excessive sedation, which is a direct factor in the development of delirium.

The incidence of adverse events in this study was $13.5 \%$, which was higher than the $6.7 \%$ reported in the previous study (15), although all events were mild (Grade 1) and did not become severe due to early response. Based on the results of the previous study and this study, a low dose of intravenous chlorpromazine is expected to be a safe treatment option for sleep disturbances in patients with a high risk of delirium.

This study has several large limitations. Firstly, because only patients referred for treatment by the palliative care team were assessed, many intractable and treatmentresistant sleep disturbance patients were included, and this study cannot be generalized due to bias in the selection of study subjects. Secondly, there is no validated subjective sleep assessment tool for patients with incurable cancer, including the SMHSQ. However, many real-world practices routinely use the SMHSQ as a tool to assess hospitalized patients. Third, this was a prospective observational study without a control cohort. Thus, common drug interactions with other medications used in hospice, such as morphine, were not investigated. This may be believed as an acceptable limitation because sleep medications or antipsychotics were not adjusted during the chlorpromazine treatment. Lastly, this study was conducted at a single institution, and future studies should use a larger dataset. Based on these limitations, this is considered a preliminary study.

\section{Conclusions}

This study indicates that intravenous chlorpromazine has a high tolerability and can be used effectively to treat sleep disturbance in patients with incurable cancer, with oral administration difficulty. Additionally, intravenous chlorpromazine is an excellent therapeutic option for sleep disturbances in patients with a high risk of delirium.

\section{Acknowledgments}

We thank the palliative care team members of the Kansai Medical University Hospital and would like to thank Enago (www.enago.jp) for the English language review.

Funding: None.

\section{Footnote}

Reporting Checklist: The authors have completed the STROBE reporting checklist. Available at https://dx.doi. org/10.21037/apm-21-948

Data Sharing Statement: Available at https://dx.doi. org/10.21037/apm-21-948

Peer Review File: Available at https://dx.doi.org/10.21037/ apm-21-948

Conflicts of Interest: All authors have completed the ICMJE uniform disclosure form (available at https://dx.doi. org/10.21037/apm-21-948). The authors have no conflicts of interest to declare.

Etbical Statement: The authors are accountable for all aspects of the work in ensuring that questions related to the accuracy or integrity of any part of the work are appropriately investigated and resolved. This study was conducted in accordance with the Declaration of Helsinki (as revised in 2013) and received approval from the Medical Ethics Committee of Kansai Medical University, Japan (reference number: 2017323). Informed consent was obtained from all study participants. This study was registered at the University Hospital Medical Information Network Clinical Trials Registry (approval number: UMIN000043869) on April 8, 2021 (registered retrospectively).

Open Access Statement: This is an Open Access article distributed in accordance with the Creative Commons Attribution-NonCommercial-NoDerivs 4.0 International License (CC BY-NC-ND 4.0), which permits the noncommercial replication and distribution of the article with the strict proviso that no changes or edits are made and the original work is properly cited (including links to both the formal publication through the relevant DOI and the license). See: https://creativecommons.org/licenses/by-nc-nd/4.0/.

\section{References}

1. Hugel H, Ellershaw JE, Cook L, et al. The prevalence, key causes and management of insomnia in palliative care patients. J Pain Symptom Manage 2004;27:316-21.

2. Mercadante S, Adile C, Ferrera P, et al. Sleep disturbances in advanced cancer patients admitted to a supportive/ 
palliative care unit. Support Care Cancer 2017;25:1301-6.

3. Davis MP, Goforth HW. Long-term and short-term effects of insomnia in cancer and effective interventions. Cancer J 2014;20:330-44.

4. Holbrook AM, Crowther R, Lotter A, et al. Meta-analysis of benzodiazepine use in the treatment of insomnia. CMAJ 2000;162:225-33.

5. Hirst A, Sloan R. Benzodiazepines and related drugs for insomnia in palliative care. Cochrane Database Syst Rev 2002;(4):CD003346.

6. Gaudreau JD, Gagnon P, Harel F, et al. Psychoactive medications and risk of delirium in hospitalized cancer patients. J Clin Oncol 2005;23:6712-8.

7. Leucht S, Cipriani A, Spineli L, et al. Comparative efficacy and tolerability of 15 antipsychotic drugs in schizophrenia: a multiple-treatments meta-analysis. Lancet 2013;382:951-62.

8. Hosie A, Davidson PM, Agar M, et al. Delirium prevalence, incidence, and implications for screening in specialist palliative care inpatient settings: a systematic review. Palliat Med 2013;27:486-98.

9. Kvale EA, Shuster JL. Sleep disturbance in supportive care of cancer: a review. J Palliat Med 2006;9:437-50.

10. Hui D, Reddy A, Palla S, et al. Neuroleptic prescription pattern for delirium in patients with advanced cancer. J Palliat Care 2011;27:141-7.

11. Hasuo H, Kanbara K, Fujii R, et al. Factors Associated with the Effectiveness of Intravenous Administration of Chlorpromazine for Delirium in Patients with Terminal Cancer. J Palliat Med 2018;21:1257-64.

12. Yeung PK, Hubbard JW, Cooper JK, et al. A study of the kinetics of chlorpromazine sulfoxide by a specific radioimmunoassay after a single oral dose of chlorpromazine in healthy volunteers. J Pharmacol Exp Ther 1983;226:833-8.

13. Yeung PK, Hubbard JW, Korchinski ED, et al. Pharmacokinetics of chlorpromazine and key metabolites. Eur J Clin Pharmacol 1993;45:563-9.

14. Kwon JH, Kim MJ, Bruera S, et al. Off-Label Medication Use in the Inpatient Palliative Care Unit. J Pain Symptom Manage 2017;54:46-54.

15. Hasuo H, Fujii R, Uchitani K, et al. Intravenous Chlorpromazine for the Short-Term Treatment of Insomnia in End-Stage Cancer Patients With Difficulty in Oral Administration. J Pain Palliat Care Pharmacother 2018;32:134-40.

16. Ellis BW, Johns MW, Lancaster R, et al. The St. Mary's Hospital sleep questionnaire: a study of reliability. Sleep 1981;4:93-7.
17. Leigh TJ, Bird HA, Hindmarch I, et al. Factor analysis of the St. Mary's Hospital Sleep Questionnaire. Sleep 1988;11:448-53.

18. Hoey LM, Fulbrook P, Douglas JA. Sleep assessment of hospitalised patients: a literature review. Int J Nurs Stud 2014;51:1281-8.

19. Yeung V, Sharpe L, Glozier N, et al. A systematic review and meta-analysis of placebo versus no treatment for insomnia symptoms. Sleep Med Rev 2018;38:17-27.

20. Chouinard G, Margolese HC. Manual for the Extrapyramidal Symptom Rating Scale (ESRS). Schizophr Res 2005;76:247-65.

21. Verkissen MN, Hjermstad MJ, Van Belle S, et al. Quality of life and symptom intensity over time in people with cancer receiving palliative care: Results from the international European Palliative Care Cancer Symptom study. PLoS One 2019;14:e0222988.

22. Sateia MJ, Buysse DJ, Krystal AD, et al. Clinical practice guideline for the pharmacologic treatment of chronic insomnia in adults: an American Academy of Sleep Medicine Clinical Practice Guideline. J Clin Sleep Med 2017;13:307-49.

23. Janno S, Holi M, Tuisku K, et al. Prevalence of neuroleptic-induced movement disorders in chronic schizophrenia inpatients. Am J Psychiatry 2004;161:160-3.

24. Hirose $S$. The causes of underdiagnosing akathisia. Schizophr Bull 2003;29:547-58.

25. Salem H, Nagpal C, Pigott T, et al. Revisiting antipsychotic-induced akathisia: current issues and prospective challenges. Curr Neuropharmacol 2017;15:789-98.

26. Hedenmalm K, Güzey C, Dahl ML, et al. Risk factors for extrapyramidal symptoms during treatment with selective serotonin reuptake inhibitors, including cytochrome P-450 enzyme, and serotonin and dopamine transporter and receptor polymorphisms. J Clin Psychopharmacol 2006;26:192-7.

27. Kondo T, Otani K, Tokinaga N, et al. Characteristics and risk factors of acute dystonia in schizophrenic patients treated with nemonapride, a selective dopamine antagonist. J Clin Psychopharmacol 1999;19:45-50.

Cite this article as: Fujii $\mathrm{R}$, Hasuo $\mathrm{H}$, Sakuma $\mathrm{H}$, Okada M, Uchitani K. The efficacy and safety of intravenous chlorpromazine treatment for sleep disturbance in patients with incurable cancer, with oral administration difficulty: a 1-week, prospective observational study. Ann Palliat Med 2021;10(8):85478556. doi: 10.21037/apm-21-948 